\title{
Representation and learning in motor action - Bridges between experimental research and cognitive robotics
}

\author{
Thomas Schack ${ }^{\mathrm{a}, \mathrm{c}, \mathrm{d}, *}$, Helge Ritter ${ }^{\mathrm{b}, \mathrm{c}, \mathrm{d}}$ \\ a Department of Neurocognition and Action Biomechanics, Bielefeld University, Bielefeld, Germany \\ ${ }^{\mathrm{b}}$ Neuroinformatics Group, Bielefeld University, Bielefeld, Germany \\ ${ }^{c}$ CITEC - Center of Excellence "Cognitive Interaction Technology", Bielefeld University, Bielefeld, Germany \\ ${ }^{\mathrm{d}}$ Research Institute for Cognition and Robotics, CoR-Lab, Bielefeld University, Bielefeld, Germany
}

\section{Keywords:}

Representation

Learning

Cognitive robotics

Interaction

Long-term-memory

Manual action

\begin{abstract}
A B S T R A C T
To gain a better understanding of the functionality of representation and categorization in action and interaction, it is fundamental that researchers understand how movements are represented in long-term memory. It is our position that human motor control requires that our actions be planned and represented in terms of intended perceptual effects and future task demands, and that the individual has a well-structured mental representation of the task so that the movement can be carried out successfully. Basic Action Concepts (BACs) are identified as major building blocks of cognitive representation in long-term memory, which are cognitive tools used to master the functional demands of movement tasks. In this paper, we consider relevant issues in research methodology and present an experimental method that can be used to assess action-relevant representational structures. This method permits us to observe the strong relationship between cognitive representation and performance in manual action. For example, the specific differences in the mental representations of participants are strongly related to skill level, as well as biomechanical and task constraints. We then discuss results from our learning experiments, where we have examined the development and changes in cognitive representation over time. From these experiments we have found that cognitive reference structures include task-specific spatial information, which provides the basis for action control in skilled voluntary movement. We have implemented these results on various robotic platforms. We argue that the insights gained from various experimental approaches in the field of cognitive psychology and motor control enable researchers to explore the possibilities and limitations of artificial control architectures in robot systems. Finally, we argue that this is not a unidirectional process. Researchers from the field of cognitive psychology and motor control can profit from the advances in technological systems, which enhance the understanding of human motor control in skilled voluntary action.
\end{abstract}

(c) 2013 Elsevier Ltd. All rights reserved.

\footnotetext{
* Corresponding author. Bielefeld University, Neurocognition and Action-Department, Center of Excellence "Cognitive Interaction Technology", Postbus 100 131, 33501 Bielefeld, Germany. Tel.: +49 5211065127.

E-mail addresses: thomas.schack@uni-bielefeld.de (T. Schack), helge@ techfak.uni-bielefeld.de (H. Ritter).
}

\section{Introduction}

In his now seminal work, "The accuracy of voluntary movement", Woodworth (1899) stated that "in all sorts of psychology, save one, there is of late an increasing interest in the motor side of consciousness... In short, the evident fact that man is not merely perceptive and intellectual, but distinctly active or reactive, is being pushed to a position in 
our study more worthy of its fundamental importance" (p. 1). When such a declaration was made more than 100 years ago it is surprising that the recent situation in psychology and motor control research can be characterized as the positive crisis of cognitive motor control research. Motor control research has received little attention and has even been called the "Cinderella of psychology" (Rosenbaum, 2005). But it may also be said motor control research has ignored higher cognitive functions to some extent and particularly neglected the interaction between different modules and levels of action organization (see Schack \& Tenenbaum, 2004a, 2004b).

Over the last 30 years, motor control research has been dominated by two perspectives: the motor approach, and the dynamic systems theory. The motor approach is primarily concerned with understanding how movements are controlled. For example, the theory of generalized motor programs (Schmidt \& Lee, 2005) suggests that the temporal and spatial muscle patterns in a movement are determined by certain parameters. Parameters deemed invariant (e.g., relative duration, relative force, muscular activation patterns) are stored in long-term memory, and are fundamental to the motor program. Parameters considered to be variant (e.g., absolute duration and absolute force) also need to be planned for motor performance, but are handled in a situation-specific way.

In contrast, the ecological or dynamical systems perspective is concerned with the basic laws of selforganization (drawn from biology and physics) and describing behavior in nonlinear dynamic terms, rather than elucidating or understanding the cognitive components of motor control. For instance, the ecological theory of affordances assumes that individuals pick up taskrelevant cues in a direct manner, and that these cues provide the actor with information for skill selection that does not necessarily rely on cognitive components, such as motor memory or perceptual categories (e.g. Gibson, 1977). In the later part of the 20th century, the dispute over these approaches was described as a paradigm crisis (Abernethy \& Sparrow, 1992); however in more recent years the debate between these two perspectives seems to have entered a stalemate, "with both sides agreeing to disagree" (Summers, 1998).

Although the motor approach and dynamic systems still dominate the field of motor control, in recent years new perspectives have emerged through which human behavior can be described and understood. The new perspectives show remarkable similarities in that they emphasize the goal-directedness of actions, the importance of anticipated perceptual effects, and the crucial role of mental representations in action control; however, they are not aligned with either side in the now-traditional debate. These are perceptual-cognitive approaches, proposing that voluntary movements are planned and performed with the help of structured cognitive representations of action effects in motor memory (Hoffmann, Butz, Herbort, Kiesel, \& Lenhard, 2007; Hommel, Muesseler, Aschersleben, \& Prinz, 2001; Mechsner, Kerzel, Knoblich, \& Prinz, 2001; Schack \& Mechsner, 2006). Furthermore, because these representations govern the tuning of motor commands and muscular activity patterns, skillful coordination occurs when appropriate mental representations of the motor task and action goals are constructed.

This perceptual-cognitive approach to movement control is reminiscent of classical ideas in psychology, such as the "ideomotor" approach adopted by Lotze (1852) and James (1890) in the 19th century or the model-theory studies of the construction of movement presented by Bernstein (1947) in the middle of the 20th century. This perspective never disappeared completely; but it was eclipsed by the dominant division of labor between the cognitive and dynamical systems approaches to motor control. More recently, there has been the suggestion that a perceptual-cognitive framework (or architecture) for motor action might fill the void left by these two dominant perspectives.

An important reason for the new interest in a cognitiveperceptual and architectural understanding of action in psychology is the impressive development of cognitive robotics. Current robotic technology has matured to the point that it can approximate a reasonable spectrum of isolated perceptual, cognitive, and motor capabilities. These advances have enabled researchers to explore how these functions might be integrated into a meaningful architecture for controlling robotic action. These cognitiveperceptual approaches also provide an opportunity to fit models on cognitive and motor related levels together with implementation architectures and simulations generated for robot actions. Because psychology traditionally relies more on phenomena-oriented theories with a narrow range of coverage than on architectural theories, cognitive robotics can help to develop a more unified, architectural understanding of human action (see Pezzulo \& Calvi, 2011; Schack, 2010). This is a new opportunity to integrate the investigation of motor control phenomena into the experimental study of action-based cognition and to address the interaction between motor and cognitive processes experimentally. Among the key issues to be addressed in this paper are how structured cognitive representations can arise during motor skill acquisition and how these underlying processes can be simulated so that they can be replicated on robotic platforms. Working toward this goal, we will translate our findings in studies of motor control and cognitive representations in humans into models that can guide the implementation of cognitive robotic architectures.

\section{Cognitive representation}

From an evolutionary and from a learning perspective, conscious cognitive functions can be assumed to emerge from more elementary functions. Whereas elementary functions (e.g., reflexes) are influenced directly by stimulus constellations, mental control functions are guided intentionally; they are regulated by the self. For example, it is not possible for a mentally controlled action to emerge from the grasp reflex in humans. In fact, this reflex has to be actively inhibited before verbal or other cognitive tools can be applied and a goal-directed action can be formed. Should children, at this point in their development, fail to develop any inhibitory activity, they will not be able to manipulate objects at all. All they will be capable of is a 
reactive but not a goal-oriented grasp. The same applies on an ontogenetically more advanced level to associations (between stimuli and action schemes) that were appropriate at one time in the past, but have now become both automated and purposeless. From such a point of view cognitive representations have to integrate and organize "lower-level" building blocks or codes (see also Perrig \& Hofer, 1989; Viviani, 1986). Within such a system or architecture, learning can be treated as a product of developing and modifying the mediating cognitive-perceptual structures in motor memory.

The cognitive-perceptual representations used in action control might involve different formats such as propositions, relational structures of many kinds, and concepts.

Researchers from different fields, such as cognitive psychology, cognitive robotics and sport psychology (Maycock et al., 2010; Schack, 2004a, 2004b; Schack \& Mechsner, 2006; Schack \& Ritter, 2009; Tenenbaum et al., 2009), have provided evidence for so-called basic action concepts (BACs) in the control of human movements. Analogous to the well-established notion of basic concepts for objects (Rosch, 1978), BACs are meant to be the mental counterparts of functionally relevant elementary components or transitional states (body postures) of complex movements. BACs are based on the cognitive chunking of body postures and movement events concerning common functions in realizing action goals. They do not refer to behavior-related invariance properties of objects, as in the case in basic object concepts, but to perception-linked invariance properties of movements.

How such BACs are integrated into higher-order representation structures has been investigated and simulated with different methods (see Krause, Dürr, Bläsing, \& Schack, 2010; Maycock et al., 2010; Schack, 2004a). Based on an experimental approach Schack and Mechsner (2006) studied the tennis serve to investigate the nature and role of long-term memory in skilled athletic performance. In high-level experts, these representational frameworks were organized in a distinctive hierarchical tree-like structure, were remarkably similar between individuals, and were well matched with the functional and biomechanical demands of the task. In comparison, action representations in low-level players and non-players were organized less hierarchically, were more variable between persons, and were less well matched with functional and biomechanical demands.

The results from a number of different activities (e.g., golf, soccer, wind surfing, volleyball, gymnastics, dancing) have demonstrated that mental representation structures are functionally related to performance and to expertise (Bläsing, Puttke, \& Schack, 2010; Bläsing, Tenenbaum, \& Schack, 2009; Schack, 2004a; Schack \& Bar-Eli, 2007; Schack \& Hackfort, 2007; Velentzas, Heinen, \& Schack, 2011).

Furthermore, as shown in a volleyball study (Schack, 2004a, 2004b), these mental representation structures are position- and thereby task-dependent. Such representation structures are the outcome of an increasing, effortreducing formation of order in long term memory (LTM). The order that has been formed is clearly related to the structure of the movement. With increasing expertise, the representation of the movement corresponds more and more to its topological (spatiotemporal) structure. Accordingly, movement control becomes possible by representing the anticipated perceptual effects of the movement and comparing them with incoming perceptual effects.

From such a point of view the structure of cognitive representations in long term memory is also relevant for perception and visuomotor control in motor action. But little is known about the relationship of cognitive representations and visuomotor control for complex movements. Therefore, we investigated the extent to which novices and skilled high-jump athletes are able to unconsciously identify visually presented body postures of the high jump. We also asked whether or not the manner of processing differs (qualitatively or quantitatively) between these groups as a function of their cognitive representation and motor expertise. An experiment with stimuli not consciously perceivable (specifically, body postures in the high jump) was designed to determine whether subliminal priming of movement phases affects target processing. Participants had to decide which phase of the high jump (approach vs. flight) a target photograph was taken from. We found a main effect of temporal order for skilled athletes, that is, faster reaction times for prime-target pairs that reflected the natural movement order as opposed to the reversed movement order. For novices, data analyses revealed an interaction between temporal order and movement phases: only the reversed movement order of flight-approach pictures increased processing time. Taken together, the results of this study suggest that the structure of cognitive movement representation modulates unconscious processing of movement pictures and points to a functional role of cognitive motor representations in visual perception (Güldenpenning, Koester, Kunde, Weigelt, \& Schack, 2011).

To learn more about different types and formats of such motor representation we studied the relationship between represented activities and the body schema (Bläsing, Schack, \& Brugger, 2010). The body schema has been described as the multimodal representation of the body that integrates somatosensory, proprioceptive, vestibular and visual information. Therefore, we investigated the mental representation of body parts and related activities in two individuals with congenitally absent limbs. One of the individuals was born without forearms and legs, and perceived phantoms of all four limbs, while the other individual was born without hands and arms and has two shortened legs with only one functionally intact foot, but has never experienced any phantom sensation. We measured cognitive action and body representations of these two individuals and compared their results to those of a group of paraplegic wheelchair athletes, and of a group of individuals with physically intact limbs. Results of the groups of physically intact subjects and the group of paraplegic subjects reflect modularity of the body schema (Haggard \& Wolpert, 2005), with separate clusters for the lower body, upper body, fingers and head. In contrast, the representation structure of the participant with congenital phantom limbs more closely resembled the structure of the matched control group, than the structure for the 
participant with congenitally absent limbs but without phantoms. The results provide evidence for a strong interaction of cognitive motor representation and cognitive body representations on a functional level. It seems that body representation and cognitive integration of perceptual body information are strongly influenced by cognitive motor representations (Bläsing, Schack, et al., 2010).

Based on these and many other studies, the main idea in our understanding of human motion is that major interfaces in the architecture of movement are cognitive in nature. Such a perspective does not view the motor system as being distinct from cognition. Instead, it considers both conscious and automatized processes of movement organization to be based functionally on cognitive representation structures that can be described with precision. This does not ignore the significance of emotional or motivational processes; it simply puts them to one side so that we can focus on the cognitive architecture of movement. In the next section we will provide some insights into the cognitive background of human grasping and in the functional role of cognitive representations in manual action.

\section{Cognitive representation and biomechanical factors in manual action}

Today, highly developed anthropoids, humans, and - to some extent - robots are able to perform manual actions. Manual actions allow for some sort of object-related organism-environment interaction and represent cognitive operations (specifically, classifications). Based on grasping movements, anthropoids can identify physically different objects as functionally equivalent tools for solving typical behavioral problems (such manipulating a screw). In anthropoids it has been possible to confirm the formation of cognitive concepts in manual action (Rensch, 1973). As current studies show, human grasping movements are cognitively represented on the basis of movement concepts (e.g., Basic Action Concepts) and build on effect-oriented target codes (in relation to space rather than to the body; Rosenbaum, Cohen, Jax, Van Der Wel, \& Weiss, 2007; Schack, 2004a; Schack \& Ritter, 2009).

Hence, the perceptual-cognitive control of arm and hand movements has become a topic of study in psychology, biomechanics, cognitive motion science, cognitive robotics, and bio-cybernetics. To understand the biomechanical and cognitive background of manual action in more detail, we established lines of research on the movement primitives of manual action, with the help of biomechanical and cognitive analyses. Because the production of manual actions is affected by such factors as biomechanical constraints (Rosenbaum, Meulenbroek, Vaughan, \& Jansen, 2001; Weigelt, Kunde, \& Prinz, 2006), we have carried out some developmental studies on the relationship between biomechanical constraints like the end-state-comfort effect and the cognitive representation of grasping postures in children (Stöckel, Hughes, \& Schack, 2012). Other studies have explored the link between semantic and motor memory, especially in the case of grasping (Weigelt, Rosenbaum, Hülshorst, \& Schack, 2009). Cognitive robotics has a strong interest in questions regarding the segmentation of arm movements, the control of robot actuators via neural networks (e.g. Self-Organizing Maps or SOM; Barreto, Araujo, \& Ritter, 2003), and the combination of learning strategies for motion primitives in grasping movements (Steil, Röthling, Haschke, \& Ritter, 2004).

To strengthen links between experimental research and robotics, we have studied cognitive representations not in isolation but as parts of a cognitive action architecture (Maycock et al., 2010; Schack \& Ritter, 2009). Such a perspective has consequences for the design of experimental studies and other lines of research. For instance, until recently motor planning has been studied in situations where participants carry out physical actions without a particular purpose or overarching movement goal. Yet, when one examines everyday life, it is apparent that our physical interactions are normally planned and executed with higher-order goals in mind. Therefore, we examined whether two previously discovered motor planning phenomena - the end-state comfort effect and motor hysteresis would hold up when the actions were carried out in the service of higher-order goals.

The end-state comfort effect is the tendency to avoid awkward postures at the end of a movement (Rosenbaum et al., 1990). This effect has been taken to support the notion that people anticipate future body states.

Motor hysteresis is the tendency of the motor system to switch from one state to another at different values depending on its history (e.g. on previous sequential movement order). The higher-order goal we chose to study was memorization. In focusing on memorization, we asked not only how motor planning is affected by the need to memorize, but also how memory performance might depend on the cognitive demands of motor planning. We asked students to retrieve cups from a column of drawers and memorize as many letters as possible from the insides of the cups. The end-state comfort effect and motor hysteresis were replicated in these conditions, indicating that the effects hold up when physical actions are carried out for the sake of a higher-order goal. However, one of the most reliable effects in memory research was eliminated, namely, recency effect. Recent items were not recalled better than items encountered earlier. This outcome was not an artifact of memory being uniformly poor, because the tendency of initial items to be recalled better than items in the middle of the sequence - the primacy effect - was obtained. The recency effect did not disappear because participants had to recall items in their correct order, for it was also eliminated when the items could be recalled in any order. These and other aspects of our results support recent claims for tighter links between basic mechanisms of perceptual-motor control and cognitive (symbolic) processing (representation of verbal information) than have been assumed in the past (Weigelt et al., 2009). The results support the idea that the interaction of cognitive and perceptual-motor components in human action works at different levels and is part of an overall architecture.

Another group of studies examined how the interaction of task constraints and cognitive representation of goalrelated grasping postures (end-state comfort) influences motor planning and execution in unimanual and bimanual grasping tasks, in both children and adult populations 
(Hughes, Haddad, Franz, Zelaznik, \& Ryu, 2011; Hughes, Reißig, \& Seegelke, 2011; Weigelt \& Schack, 2010). The results from these studies have shown that task constraints do not influence motor planning and motor execution equally. For example, Hughes, Haddard, et al. (2011) examined how physically connecting two objects might influence bimanual grasping and placing movements. They found that although object end-orientation congruency influences both the grasping and transport components of the task, physically connecting the two objects altered only the degree of interlimb coupling between the hands (e.g., on a kinematic level).

Hughes, Haddard, et al. (2011) postulate that successful task performance is contingent on the action goals of the task, which guide the selection of lower level action features (such as initial grasp postures), as well as the manner in which the task is performed (e.g., its kinematics). In particular, grasping and placing movements can be separated into an initial grasp and a transport component, within which there are a number of constraints that the system seeks to satisfy. Taken together, these results indicate that constraints may not exert equal effects on grasping and transport components, and a more holistic approach to human object manipulation may provide insights that might not be apparent otherwise.

A further line of studies focuses on the cognitive background of motor performance in manual action (Schack \& Ritter, 2009), particularly during the rehabilitation of hand function after a stroke (Braun et al., 2007, 2008). In these studies we learned that central costs and interference in manual actions depend solely on how these movements are represented on a cognitive level.

Our recent research starts with the question of how structures of sensory-motor representation are established and changed step by step in compliance with task constraints. To examine how these constraints interact with one another during manual actions in human beings and robots, we first investigated the development of structured representation (action templates) in human skill acquisition, then applied these results to robots. For instance, to learn in detail about anticipation and motor control in children, we investigated the development of the end-state comfort effect in young children aged 3, 4, and 5 (Weigelt \& Schack, 2010). The children performed a dowel placing task, reaching for a horizontal dowel and inserting one of its ends into a target disc. Although all children, regardless of age, reached for the dowel with an overhand grasp when this resulted in a comfortable end-state (i.e. thumb-up posture), a different pattern emerged when an underhand grip had to be selected. In this latter situation, only $18 \%$ of the 3 -year-olds, $45 \%$ of the 4 -year-olds, and $67 \%$ of the 5 year-olds used an underhand grip and finished the action in a comfortable end-state. These results show a distinct pattern of gradual improvement in children's sensitivity to end-state comfort across the three age-groups. Such information about basic principles of end-state comfort planning in humans is of interest when designing valid robot architectures that are used to perform manual actions and interact with human beings in an appropriate, agedependent manner (Gienger, Toussaint, Jetchev, Bendig, \& Goerick, 2008).
In a recent study we investigated anticipatory motor planning and cognitive representation of grasp postures in children aged 7, 8, and 9 years. Overall, 9-year-old children were more likely than 7 or 8 -year-olds to plan their movements to end in comfortable postures, and to have distinct representational structures for certain grasp postures. Additionally, sensitivity to comfortable end-states was related to the mental representation of certain grasp postures. Children with functionally well structured representations related to grasp comfort were more likely to have satisfied end-state comfort in both the simple and the advanced planning condition. In contrast, children whose cognitive representations were not structured by grasp comfort achieved far less satisfaction with grasp comfort in the advance planning condition. The results of the present study support the notion that cognitive action representation plays an important role in the planning and control of grasp postures (Stöckel et al., 2012).

The results of these studies show that dexterity in manual action and task related object manipulation is accompanied by order formation in memory. Such order formation in action knowledge reduces the cognitive effort required to activate relevant information. From this perspective, we have to solve movement tasks purposefully and in a step by step fashion within the framework of voluntary organization of movement. So it is of interest to learn about the task-related order formation of action knowledge. We will provide an example concerning the representation of objects with regard to manual actions in an automobile mechanic's workshop. The question is how strong a relationship we will find between the representation of object concepts (parts of a car) and the manual expertise of mechanics in a workshop.

In line with our assumptions about BACs and the structure and dimensioning (feature assignment) of action representation (Schack \& Ritter, 2009), we developed the structure-dimensional analysis (SDA-M) of mental representations (Schack, 2012). The SDA-M presents the structure-dimensional relations of conceptually ordered knowledge; psychometrically, for both single cases and groups. The SDA method proceeds in four steps. First, the splitting procedure involving the multiple sorting tasks delivers a scaling of the distances among the BACs in the predetermined set (i.e., verbal lists). Second, a hierarchical cluster analysis transforms each set of BACs into a hierarchical structure. Third, a factor analysis reveals the dimensions in this structured set of BACs; and fourth, the cluster solutions are tested for invariance within and between groups. (Psychometric details of the full procedure are provided by Schack, 2012.) For the present study, only the first two steps are relevant:

1. Because it can be assumed that the structure of cognitive representations can only be explicated to a limited extent, the scaling of distance between concepts is done with a special splitting technique. It is based on the selection and presentation of a group of concepts that are a valid component of that set of concepts that is absolutely necessary for a certain problem-solving or working domain. As in the methods mentioned above, this group of concepts is initially identified through work analysis, 
surveys, or experiments. This can be illustrated with an example of research: For the work of an automobile mechanic, we found these car parts as object concepts related to manual action in the repair shop: (1) piston; (2) cylinder; (3) valve; (4) cylinder head; (5) drive shaft; (6) contact breaker; (7) ignition coil; (8) spark plug; (9) distributor; (10) wheel mounting; (11) shock absorber; (12) disc brake; (13) coil spring; and (14) leaf spring.

The experimental procedure took the $N$ elements (in this case, 14) from the set of concepts and selected one as an anchor to which the other $N-1$ elements were assigned or not assigned, according to an individually given similarity criterion. This procedure (while retaining the original anchor) was repeated with each new positive or negative subset until either there were just indivisible sets with one object, or an individually selected break-off criterion was attained at which the sets should not be broken down further. Because each concept took the position of anchor once, we obtained a total of $N$ (14) decision trees whose nodes contained the subsets produced and whose edges had a negative or positive sign depending on whether the elements were assigned to the anchor concept.

2. The structured relations among the $N$ concepts were obtained by compiling a distance matrix from the scaling procedure and subjecting it to a hierarchical cluster analysis.

Custom computer programs were developed to apply this method so that such experiments could be carried out within a reasonable time (10-15 min; Schack, 2012). Using the SDA method presented above, we compared the action knowledge of five mechanics working in a renowned workshop (Daimler-Benz, BMW, Cologne) with the knowledge of four sports students who had driver's licenses and automobiles but no relevant experience in repairing them.

When repairing automobiles, mechanics continuously have to solve problems that require a sound knowledge of the vehicle's major subsystems (electrical system, chassis, engine) and the functions of their corresponding parts. When repairing an engine, a mechanic has to discriminate very clearly, even when just looking, between spare parts that are relevant to the current job and any irrelevant parts that may also lie within reach. The problem solving process involved in diagnosing the damage to the vehicle and how to repair it also requires functionally applicable knowledge of systems and parts as well as the appropriate tools. We found that the experts in this domain seemed to achieve this effortlessly. However, when asked, they could not report which sort of knowledge had enabled them to perform this real-time problem solving or how their knowledge was structured. Hence, we used a survey of another group of experts $(n=3)$ and a functional analysis of automobile construction to develop the list of concepts reported above.

Concepts 1-5 refer to parts of the engine (and drive train), Concepts 6-9 to the electrical system, and Concepts 10-14 to the chassis. These concepts were read into a computer program, and participants performed the aforementioned split procedure. For all concepts, participants had to decide whether or not the concepts were linked together functionally for carrying out actions during their work. Concepts were presented in random order.

One major issue in the analysis was whether the degree of structuring would be greater in the experts' than in the laypersons' action knowledge. Another was which features would be used to structure the concepts in memory. Representative results of the hierarchical cluster analysis from one expert are shown in Fig. 1.

As Fig. 1 shows, the expert has a strong hierarchical knowledge structure with three concept clusters. Such structuring points to a high degree of order formation in the expert's action knowledge. The content of this knowledge structure is highly interpretable: Each cluster refers to a specific subsystem of the vehicle: these cognitive substructures, which refer to the representation units (concepts) of the engine, electrical system, and chassis, are highly distinct. As the results of the factor analysis show, the features of these concepts are linked to problemsolving processes within these action systems (engine, electrical system, chassis). Hence, functional features of the concepts determine the structure. The expert predominantly knows for what (for what purpose) the single parts can be used. The results of this analysis will now be compared with findings for a low performer (Fig. 2).

The knowledge structure depicted in Fig. 2 reveals poor performance in the automobile repair domain. According to self-reports and a test of automobile repair skills, this person is only able to change a wheel. Compared with what is shown Fig. 1, the knowledge system for the 14 concepts in Fig. 2 is broadly unstructured. One cluster refers to the chassis; this knowledge substructure seems to be due to frequent wheel changes. Further substructures cannot be recognized in Fig. 2. This also means that no structured knowledge is available for other performance domains of automobile repair (engine, electrical system).

This study shows clearly that performance is related to the structuring of action knowledge. A comparable systematic structuring was found in all five experts. It is clear that the structure is also functionally relevant, and the performance ascertained is functionally efficient for realtime problem solving in the field of automobile repair. Such problem-solving processes are linked continuously to specific movements, routines, and habits. Therefore, it can be assumed that specific movement knowledge is also attached to this object knowledge. The relation between movement representation and learning will now be studied and discussed with an example from golf playing.

\section{Mental representations and learning in complex movements}

Mental (action) representation plays a central role controlling and implementing actions. These processes include storing the cognitive-perceptual outcomes of learning processes as items in long-term memory. From this point of view learning is nothing more than the modification and adaptation of representation structures in memory. 


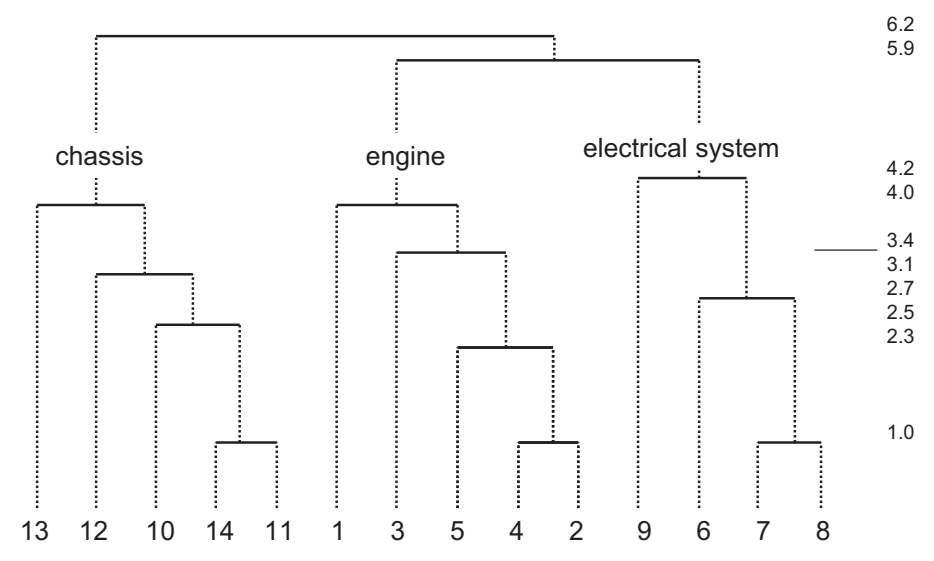

(1) Piston, (2) cylinder; (3) valve; (4) cylinder head; (5) drive shaft; (6) contact breaker; (7)

ignition coil; (8) spark plug; (9) distributor; (10) wheel mounting; (11) shock absorber; (12)

disc brake: (13) coil spring; (14) leaf spring.

Fig. 1. Results of the hierarchical cluster analysis of object concepts for a high-performance (expert) mechanic. The lower the value of a cross-connection between the study units (see the Euclidean distance scale on the right), the shorter the distance between the concepts in long-term memory ( $\alpha=.05$, dcrit $=3.42$ ). dcrit is the random critical distance between the concepts for a given $\alpha$ probability.

To understand the relationship between memory structures and learning in more detail we designed a learning study for golf (Frank, Land, \& Schack, 2013). Putting was chosen as the relevant task. The basic action concepts of the putt are: (1) shoulders parallel to target line: (2) align club face square to target line: (3) check grip; (4) look to the hole; (5) rotate shoulders away from the ball; (6) keep arms-shoulder triangle; (7) make a smooth transition; (8) rotate shoulders toward the ball; (9) accelerate club; (10) hit the ball; (11) keep club face square to target line at impact; (12) follow through; (13) rotate shoulders through the ball; (14) decelerate club; (15) direct club head to planned position; and (16) look to the outcome. Participants were instructed to sort the list of basic action concepts according to their functional relevance during movement execution. A hierarchical cluster analysis then revealed individual and group mental representation structures (for further information on the SDA-M, see Schack, 2012). For the experiment, novice golfers were randomly assigned to either a practice group $(n=12)$ or a control group $(n=12)$. Both groups were tested before and after an acquisition phase of three days as well as after a

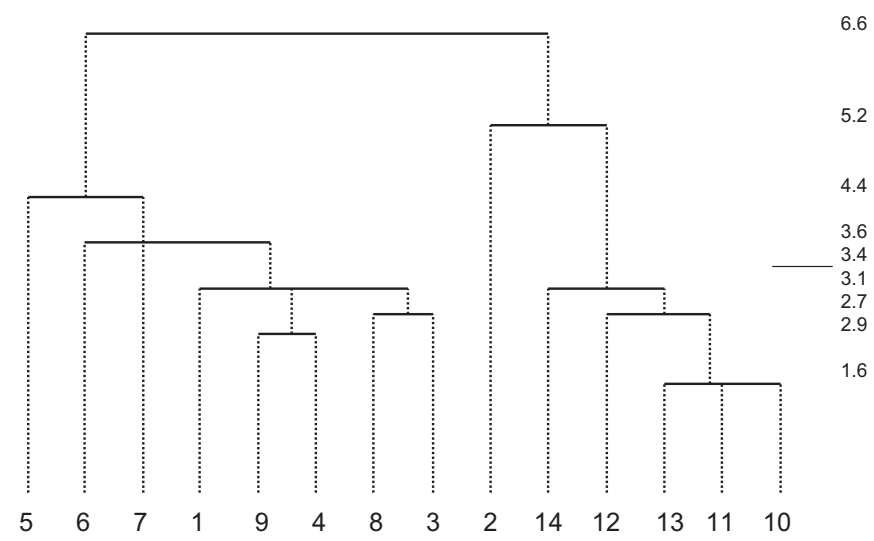

(1) Piston, (2) cylinder; (3) valve; (4) cylinder head; (5) drive shaft; (6) contact breaker; (7) ignition coil; (8) spark plug; (9) distributor; (10) wheel mounting; (11) shock absorber; (12) disc brake: (13) coil spring; (14) leaf spring.

Fig. 2. Results of hierarchical cluster analysis for a low performer (layperson) in mechanics. 
three day retention interval. All participants in both groups completed the sorting task on three occasions: day one (the pretest), day five (the posttest), and day six (the retention test). Additionally, the experimental group performed 3 blocks of 20 putts each on day one (pretest), day five (posttest) and day six (retention test). On days two, three and four, the experimental group practiced the putting task, which consisted of 10 blocks of 20 putts each with a short break between every two blocks. No feedback on technical issues was given during putting, just the outcome. The control group did not practice during this time.

Preliminary results (see Fig. 3) show changes in the experimental group's mental representation structure for the putt: the representational structures during the pretest showed no clustering of basic action concepts in the group's mean dendrogram, whereas both the posttest and the retention test revealed changes in average mental representation structure. Specifically, the experimental group's mean dendrogram displayed several clusters that already point toward the functional structure of the movement, which illustrates the development of functional structure formation in memory. Because there was no learning phase between posttest and retention test, the cognitive structure of the individuals in the posttest is statistically equivalent to the representation structure in the retention test (on results of the invariance measure).

Meanwhile, the mental representation structure for the control group did not reveal any changes between the preand post-test, which both showed no clustering whatsoever of basic action concepts in the group's mean dendrogram. In addition, while retention test for the control group revealed a few clusters, these did not correspond meaningfully to the functional demands of the task.

To learn about the relationship between cognitive representation and performance in interaction we combined an experiment with human learners and an interaction scenario between human users and a technical platform (offering a virtual human teacher). This study is presented in the next section.

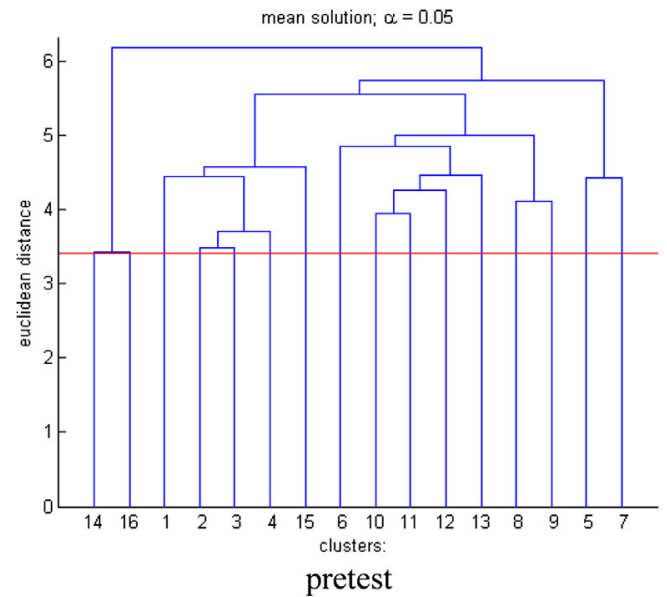

\section{Co-representation and joint attention in learning}

To study the function of cognitive representation in learning we used a new approach to study, as an initial effort, the development of cognitive representations in the context of interactions between an expert and novices in a necktie-tying task. Based on previous research suggesting that instructions about action sequences are better memorized when they are offered with appropriate gestures, we combined the motor learning task (tying the necktie) with the demonstration of gestures. Over a threeweek learning period we measured the development of mental representations prior to the experiment (pretest), after eight sessions (in the middle of the second week), after 15 sessions (at the end of the third week) and in a retention test (one week after the posttest). Subsequently, we implemented the results about the development of cognitive representations in interaction to a technical platform with a virtual agent. The participants have been students. They didn't know how to perform this particular kind of necktie tying. An expert demonstrated the movement and used verbal instructions and corrections to support the motor performance of the subjects. Analyzing cognitive representation with the SDA-M method, we defined the following 13 units as relevant action sequences or body postures.

1. Put the tie around the back of your neck, broad end on the right.

2. Broad end is hanging farther down on the right.

3. Left hand holds narrow end in front of the upper body.

4. Right hand crosses broad end over the narrow end to the left.

5. Change grasp - pull back to the right.

6. Left hand grabs half-formed knot.

7. Right hand pulls the broad end over, then underneath the outer layer of the loop around the neck.

8. Wrap the broad end all the way around the narrower end.

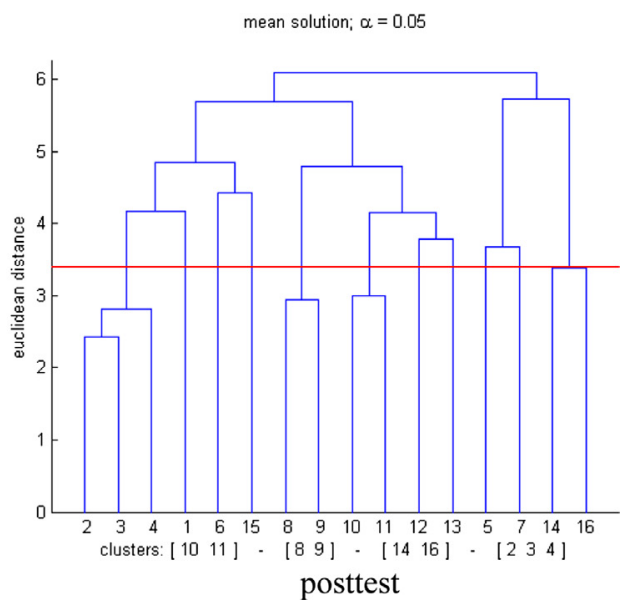

Fig. 3. Measured mental representations for putting averaged across the first ten subjects (preliminary results) of the experimental group. On the left side the results of the pretest are shown. No substructures (clusters) are statistically identified (below the critical value of 3.4). On the right side the results of the posttest are provided. The dendrogram for the posttest indicates four different clusters. $N=10, \alpha=.05$, dcrit $=3.41$. 
9. Left thumb and index finger grab newly formed knot.

10. Right hand takes broad end and places it on fingers of the left hand.

11. Pass the broad end up from below, behind the knot, through the loop around your neck.

12. Lean your head back.

13. Put the broad end underneath the outer layer of the knot and tighten up the necktie.

Over the course of the three-week intervention, participants became faster and more accurate in their performance and were more successful at completing the task after more trials. As can be seen in Fig. 4, the BACs for the action sequence are more functionally clustered in the posttest than they were in the pretest.

The cognitive representations in the post-test showed that participants divide the BACs into four functional segments, whereas action representations prior to the experiment (pretest) are less hierarchically organized and show fewer functional segments. Furthermore, the previously singled out BACs observed in the pretest are now integrated into the mental representation structure in the posttest.

As a follow-up we implemented this cognitive interaction scenario on a technical platform. The virtual human being MAX (Lessmann, Wachsmuth, \& Kopp, 2006) is capable of integrating instructions with self-generated gestures, promoting the formation of memory representations by the human listener. The quality of these representations (structure, validity) is assessed by MAX (using the SDA-M Method), yielding a measure of the listener's comprehension, which can in turn be used by MAX to adjust its future use of particular instructions and gestures in a closed-loop scenario (Fig. 5).

The main research questions are: 1) how well will a human learner acquire action sequences taught by MAX; and 2) will MAX learn to adjust his instructions based on the feedback provided through measuring the structure of the user's mental representation? After completing this study about co-representation and learning in interaction we plan to implement specific instructional routines into MAX that take differences in the user's gender, age or cultural background into account (Steggemann, Weigelt, Kopp, Vogel, \& Schack, 2011).

\section{Representations in robot architectures}

Cognitive representations do not work in isolation: control strategies, representations, and primitives chosen at the "lower levels" provide the basis on which the higher levels are constructed and strongly shape the problems to be solved at those higher levels. There is a significant body of literature focusing on selected aspects of representations for motor actions, such as contact formation, grasp optimization, and finger gaits. But it is relatively little known about the integration of these isolated aspects into an overall, integrated architecture. A promising path toward such an architecture marries recent methods and concepts from cognitive psychology, for studying cognitive representations of action, with ideas from cognitive robotics, about integrating a rich set of skills in a systematic and manageable fashion.

The case of cognitive human action control is a superb working example of such an architecture, realized under constraints that appear formidable from any engineering standpoint. Different lines of development in cognitive robotics (Maycock et al., 2010) have exposed interesting parallels to a cognitively motivated architecture for human motor action (Schack, 2004a, 2004b; Schack \& Ritter, 2009). This architecture addresses cognitive representations as a part of an action system, postulating four levels: a sensorimotor level providing an interface to sensors and effectors, two intermediate levels of sensorimotor and mental representations, accommodating basic action units at different levels of abstraction, and a topmost level of mental control shaping our purposeful behavior (see Fig. 6). This model has been our rationale for establishing a research environment for studying manual interactions. This enables us to explore correspondences between the computational manual action architecture on the one side, and the sensorimotor and mental representations levels of the cognitive model on the other (see Fig. 6), and to bring to bear methods from both disciplines to mutually refine and cross-connect the two accounts, with the aim of producing a coherent and overarching picture of complex action and especially of manual intelligence (see Maycock et al., 2010).

To bridge the gap between empirical research and robotic design, we have to translate our findings from studies of human movement into models sufficiently specific to

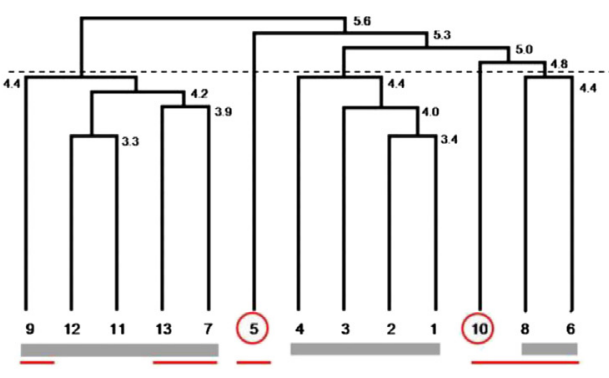

Pretest

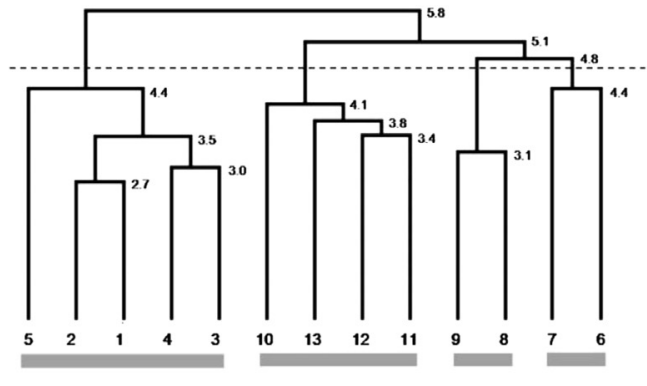

Posttest

Fig. 4. Adaptation of memory structures while learning to tie a necktie. On the pretest the dendrogram indicates a low-level representation of the activity. Some elements $(5,10)$ are not included in the representation structure, which reflects the task structure (action sequences) only to low degree. In the posttest the dendrogram indicates a task-oriented cognitive representation of the movement elements, $N=9 ; \alpha=.01 ;$ dcrit $=4.59$. 


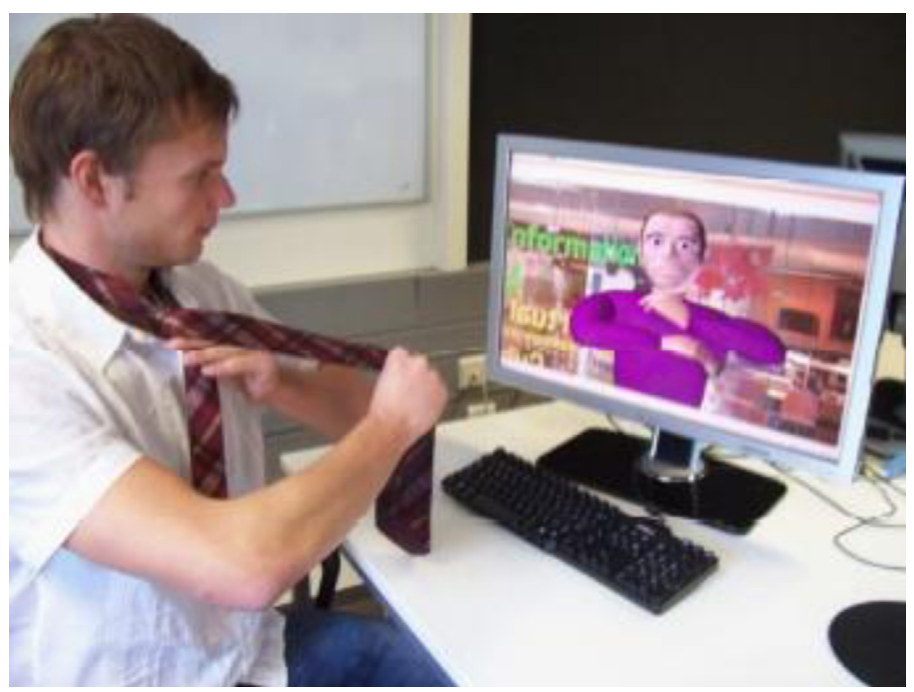

Fig. 5. In a teacher-trainee scenario, the virtual agent MAX supervises the acquisition of short action sequences (e.g., tying a necktie) by verbally instructing participants, signaling the movements with gestures, and assessing changes of mental representation during skill learning to provide feedback.

permit an implementation in a robotic system. On the robotics side, we use several layers of representations, ranging upward from specialized controllers at the lowest level, to basis postures defining set-points for the controllers, to manifolds that interpolate between basis postures, to hierarchical state machines to switch between different interaction regimes and, finally, to declarative, humanreadable XML specifications for the topmost level of system structure. As part of a multilevel research strategy, we have examined the hierarchical representation of objects under different task constraints, and, complementary to this, the hierarchical representation of grasping movements between power and precision grips (Bläsing, Puttke, et al., 2010; Bläsing, Schack, et al., 2010). Studies on the cognitive representation of movement primitives (in the form of BACs) helped us to further differentiate the taxonomy of grasps for which the robot hand is equipped. In another research line we captured motion data for basis hand postures in various grasping actions (Maycock et al., 2010). Using small set of prototypical hand postures as pre-grasps, we developed a robust method for grasping a wide range of household objects (Röthling, Steil, \& Ritter, 2007). This approach offers interesting cross-connections with the concept of basic action units (BACs) and the results of the studies previously presented. In the studies concerning the representation of golf putting, necktie tying, or manual actions we learned that BACs are integrated into hierarchies, whose structure can be extracted with specialized experimental methods (SDA-M), allowing to further examine how these representations change during learning. The insights gained in such experiments have guided the implementation of computational strategies on robotic platforms (i.e., a 7 degree of freedom robot arm setup; see Ritter, Steil, Noelker, Roethling, \& McGuire, 2003; Schack \& Ritter, 2009).

Insights gained from the attempt to validate the hypothesis about action and representation structures in robot learning can be used strategically to inform the design of experiments with human subjects. For instance, to improve grasping by the robot hand and to learn about the "granularity" of cognitive building blocks in manual actions, we conducted experiments to gain more detailed insight into the relationship between the structure of representations and the performance of manual actions, including situations in which the actions result in error (Bläsing, Puttke, et al., 2010; Bläsing, Schack, et al., 2010; Schack \& Ritter, 2009).

Connecting research into the cognitive architectures of human beings and robots are the cognitive benchmarks (hierarchical representations) for manual actions, especially

\begin{tabular}{|lr|}
\hline Mental Control & XML-based Memory Layer \\
Mental Representations & Hierarchical State Machines \\
\hline Sensorimotor Representations & Manifolds Basis Postures- \\
\hline Sensorimotor Control & Low-Level Controllers \\
\hline
\end{tabular}

Fig. 6. A tentative integration of cognitive (mental) representations in the architecture of action. The left side indicates cognitively motivated levels; the right indicates possible implementations in a technical system. The figure is taken from Maycock et al. (2010). 
the representation of objects and grasping postures. The kind of integrated (interdisciplinary) research that has been described here allows us to explore experimentally the interactions between action representation in memory (as simulated with different kinds of artificial neural networks) and motor skills in the context of real-world tasks (Krause et al., 2009). The present studies of the mental representation of grasping postures in humans and other experimental studies with the robot hand (Schack \& Ritter, 2009) will better enable us to make step by step refinements in robot hand grasping.

\section{References}

Abernethy, B., \& Sparrow, W. A. (1992). The rise and fall of dominant paradigms in motor behavior research. In J. J. Summers (Ed.), Approaches to the study of motor control and learning (pp. 3-45). Amsterdam: Elsevier Science.

Barreto, G., Araujo, A., \& Ritter, H. (2003). Self-organizing feature maps for modeling and control of robotic manipulators. Journal of Intelligent and Robotic Systems, 24, 407-450.

Bernstein (Bernštejn), N. A. (1947). O postrojenii dviženij [The construction of movements]. Moskva: Medgiz.

Bläsing, B., Puttke, M., \& Schack, T. (Eds.). (2010). Neurocognition of dance). London: Psychology Press.

Bläsing, B., Schack, T., \& Brugger, P. (2010). The functional architecture of the human body: assessing body representation by sorting body parts and activities. Experimental Brain Research, 203, 119-129.

Bläsing, B., Tenenbaum, G., \& Schack, T. (2009). The cognitive structure of movements in classical dance. Psychology of Sport and Exercise, 10(1), 350-360.

Braun, S. M., Beurskens, A. J. H. M., Schack, T., Marcellis, R. G., Oti, K. C., Schols, J. M., et al. (2007). Is it possible to use the SDA-M to investigate representations of motor actions in stroke patients? Clinical Rehabilitation, 21, 822-832.

Braun, S. M., Kleynen, M., Schols, J. M., Schack, T., Beurskens, A. J., \& Wade, D. T. (2008). Using mental practice in stroke rehabilitation: a framework. Clinical Rehabilitation, 7, 579-591.

Frank, C., Land, W. M., \& Schack, T. (2013). Mental representation and learning: the influence of practice on the development of mental representation structure in complex action. Psychology of Sport and Exercise, 14, 353-361.

Gibson, J. J. (1977). The theory of affordances. In R. Shaw, \& J. Bransford (Eds.), Perceiving, acting, and knowing: Toward an ecological psychology (pp. 67-82). Hillsdale, NJ: Erlbaum.

Gienger, M., Toussaint, M., Jetchev, N., Bendig, A., \& Goerick, C. (2008). Optimization of fluent approach and grasp motions. 8th IEEE-RAS International Conference on Humanoid Robots.

Güldenpenning, I., Koester, D., Kunde, W., Weigelt, M., \& Schack, T. (2011). Motor expertise modulates the unconscious processing of human body postures. Experimental Brain Research, 213(4), 383-391.

Haggard, P., \& Wolpert, D. M. (2005). Disorders of body scheme. In H.J. Freund, M. Jeannerod, \& M. Hallett (Eds.), Higher-order motor disorders: From neuroanatomy and neurobiology to clinical neurology (pp. 261-272). Oxford: Oxford University Press.

Hoffmann, J., Butz, M. V., Herbort, O., Kiesel, A., \& Lenhard, A. (2007). Spekulationen zur Struktur ideo-motorischer Beziehungen. Zeitschrift für Sportpsychologie, 14, 95-104.

Hommel, B., Muesseler, J., Aschersleben, G., \& Prinz, W. (2001). The Theory of Event Coding (TEC): a framework for perception and action planning. Behavioral and Brain Sciences, 24(5), 849-878.

Hughes, C. M. L., Haddad, J. M., Franz, E. A., Zelaznik, H. N., \& Ryu, J. H. (2011). Physically coupling two objects in a bimanual task alters kinematics but not end-state comfort. Experimental Brain Research, 211(2), 219-229.

Hughes, C. M. L., Reißig, P., \& Seegelke, C. (2011). Motor planning and execution in left and right-handed individuals during a bimanual grasping and placing task. Acta Psychologica, 138, 111-118.

James, W. (1890)The principles of psychology, Vol. 1, New York: Holt.

Krause, A. F., Bläsing, B., Dürr, V., \& Schack, T. (2009). Direct control of an active tactile sensor using echo state networks. In H. Ritter, G. Sagerer, R. Dillmann, \& M. Buss (Eds.), Human Centered Robot Systems. Cognition, Interaction, Technology (pp. 11-21). Berlin Heidelberg: Springer-Verlag.
Krause, A. F., Dürr, V., Bläsing, B., \& Schack, T. (2010). Multiobjective optimization of echo state networks for multiple motor pattern learning. In R. Tetzlaff, W. Schwarz, et al. (Eds.), 18th IEEE workshop on nonlinear dynamics of electronic systems (NDES 2010) (pp. 190-193).

Lessmann, N., Kopp, S., \& Wachsmuth, I. (2006). Situated interaction with a virtual human - perception, action, and cognition. In G. Rickheit, \& I. Wachsmuth (Eds.), Situated communication (pp. 287-323). Berlin: Mouton de Gruyter.

Lotze, R. H. (1852). Medizinische Psychologie oder Physiologie der Seele (pp. 287-325). Leipzig, Germany: Weidmann'sche Buchhandlung.

Maycock, J., Dornbusch, D., Elbrechter, C., Haschke, R., Schack, T., \& Ritter, H. (2010). Approaching manual intelligence. Künstliche Intelligenz, . http://dx.doi.org/10.1007/s13218-010-0064-9. http://www. springerlink.com/content/tgv61350px27q482.

Mechsner, F., Kerzel, D., Knoblich, G., \& Prinz, W. (2001). Perceptual basis of bimanual coordination. Nature, 414, 69-72.

Perrig, W. J., \& Hofer, D. (1989). Sensory and conceptual representations in memory: motor images that cannot be imaged. Psychological Research, 51, 201-207.

Pezzulo, G., \& Calvi, G. (2011). Computational explorations of perceptual symbol system theory. New Ideas in Psychology.

Rensch, B. (1973). Handgebrauch und Verständigung bei Affen und Frühmenschen. Bern: Huber.

Ritter, H., Steil, J. J., Noelker, C., Roethling, F., \& McGuire, P. (2003). Neural architectures for robotic intelligence. Reviews in the Neurosciences, 14(1-2), 121-143.

Rosch, E. (1978). Principles of categorization. In E. Rosch, \& B. B. Lloyd (Eds.), Cognition and categorization (pp. 27-48). Hillsdale, NJ: Erlbaum.

Rosenbaum, D. A. (2005). The Cinderella of psychology: the neglect of motor control in the science of mental life and behavior. American Psychology, 60(4), 308-317.

Rosenbaum, D. A., Cohen, R. G., Jax, S. A., Van Der Wel, R., \& Weiss, D. J. (2007). The problem of serial order in behavior: Lashley's legacy. Human Movement Science, 26, 525-554.

Rosenbaum, D. A., Meulenbroek, R. G., Vaughan, J., \& Jansen, C. (2001). Posture-based motion planning: applications to grasping. Psychological Review, 108(4), 709-734.

Rosenbaum, D. A., Marchak, F., Barnes, H. J., Vaughan, J., Slotta, J., \& Jorgensen, M. (1990). Constraints for action selection: Overhand versus underhand grips. In M. Jeannerod (Ed.), Attention and Performance XIII: Motor representation and control (pp. 321-342). Hillsdale, NJ: Lawrence Erlbaum Associates.

Röthling, F., Haschke, R., Steil, J. J., \& Ritter, H. (2007). Platform portable anthropomorphic brasping with the Bielefeld 20 DOF Shadow and 9 DOF TUM Hand. IEEE IROS Conference Proceedings, pp. 2951-2956.

Schack, T. (2004a). The cognitive architecture of complex movement. Special Issue Part II: the construction of action - new perspectives in movement science. International Journal of Sport and Exercise Psychology, 2(4), 403-438.

Schack, T. (2004b). Knowledge and performance in action. Journal of Knowledge Management, 8(4), 38-53.

Schack, T. (2010). Die kognitive Architektur menschlicher Bewegungen Innovative Zugänge für Psychologie, Sportwissenschaft und Robotik. Reihe "Sportforum". Aachen: Meyer \& Meyer.

Schack, T. (2012). A method for measuring mental representation. In G. Tenenbaum, \& B. Eklund (Eds.), Handbook of measurement in sport (pp. 203-214). Champaign, Illinois: Human Kinetics.

Schack, T., \& Bar-Eli, M. (2007). Psychological factors in technical preparation. In B. Blumenstein, R. Lidor, \& G. Tenenbaum (Eds.), Psychology of sport training (pp. 62-103). Oxford: Meyer \& Meyer Sport.

Schack, T., \& Hackfort, D. (2007). An action theory approach to applied sport psychology. In G. Tenenbaum, \& R. C. Eklund (Eds.), Handbook of sport psychology (3rd ed.). (pp. 332-351) NJ: Wiley.

Schack, T., \& Mechsner, F. (2006). Representation of motor skills in human long-term memory. Neuroscience Letters, 391, 77-81.

Schack, T., \& Ritter, H. (2009). The cognitive nature of action - functional links between cognitive psychology, movement science and robotics. In M. Raab, J. Johnson, \& H. Heukeren (Eds.), Progress in brain research: Mind and motion - The bidirectional link between thought and action (pp. 231-252). Amsterdam: Elsevier.

Schack, T., \& Tenenbaum, G. (2004a). The construction of action - new perspectives in science. Special Issue Part I: perceptual and cognitive control. International Journal of Sport and Exercise Psychology, 2(3), 207-209.

Schack, T., \& Tenenbaum, G. (2004b). The construction of action - new perspectives in science. Special Issue Part II: representation and planning. International Journal of Sport and Exercise Psychology, 2(4), 343-345. 
Schmidt, R. A., \& Lee, T. D. (2005). Motor control and learning: A behavioral emphasis. Champaign, IL: Human Kinetics.

Steggemann, Y., Weigelt, M., Kopp, S., Vogel, L., \& Schack, T. (2011). Representation and learning in interaction with virtual agents. CITECProject. Bielefeld, Germany: Bielefeld University.

Steil, J., Roetling, F., Haschke, R., \& Ritter, H. (2004). Situated robot learning for multi-modal instruction and imitation of grasping. Robotics and Autonomous Systems, Special Issue on " Robot Learning by Demonstration", 47, pp. 129-141.

Stöckel, T., Hughes, C., \& Schack, T. (2012). Representation of grasp postures and anticipatory motor planning in children. Psychological Research, 76(6), 768-776.

Summers, J. J. (1998). Has ecological psychology delivered what it promised? In J. P. Piek (Ed.), Motor behavior and human skill - A multidisciplinary approach) Champaign, IL: Human Kinetics.

Tenenbaum, G., Hatfield, B., Eklund, R. C., Land, W. M., Calmeiro, L., Razon, S., et al. (2009). A conceptual framework for studying emotions-cognitions-performance linkage under conditions that vary in perceived pressure. In M. Raab, J. Johnson, \& H. Heukeren (Eds.), Progress in brain research (pp. 159-178). Amsterdam: Elsevier

Viviani, P. (1986). Do units of motor action really exist? Experimental Brain Research, 15, 201-215.

Velentzas, K., Heinen, T., \& Schack, T. (2011). Routine integration strategies and their effects on volleyball serve performance and players' movement mental representation. Journal of Applied Sport Psychology, 23(2), 209-222.

Weigelt, M., Kunde, W., \& Prinz, W. (2006). End-state comfort in bimanual object manipulation. Experimental Psychology, 53(2), 143-148.

Weigelt, M., Rosenbaum, D. A., Hülshorst, S., \& Schack, T. (2009). Moving and memorizing: motor planning modulates the recency effect in serial and free recall. Acta Psychologica, 132, 68-79.

Weigelt, M., \& Schack, T. (2010). The development of end-state comfort planning in preschool children. Experimental Psychology, 6, 1-7.

Woodworth, R. S. (1899). The accuracy of voluntary movement. Psychological Review, 3, 1-119. 